\title{
PORO-MECHANICAL ANALYSIS OF A BIOMIMETIC SCAFFOLD FOR OSTEOCHONDRAL DEFECTS
}

\author{
Pietro Mascheroni ${ }^{1}$, Alfio Grillo ${ }^{2}$, and Daniela P. Boso ${ }^{3}$ \\ ${ }^{1}$ Department of Systems Immunology, Helmholtz Centre for Infection Research \\ Inhoffenstraße 7, D-38124, Braunschweig, Germany \\ e-mail: pietro.mascheroni@helmholtz-hzi.de \\ ${ }^{2}$ Dipartimento di Scienze Matematiche "G.L. Lagranage", "Dipartimento di Eccellenza 2018-2002", \\ Politecnico di Torino \\ Corso Duca degli Abruzzi 24, I-10129, Torino, Italy \\ alfio.grillo@polito.it \\ ${ }^{3}$ Department of Civil, Environmental and Architectural Engineering, University of Padova \\ Via F. Marzolo 9, I-35131, Padova, Italy \\ daniela.boso@unipd.it
}

Keywords: Osteochondral defects, Tissue engineering, Biomimetic scaffolds, Porous media mechanics, Finite Element Analysis.

\begin{abstract}
Osteochondral defects are focal areas of damage involving articular cartilage and sub-chondral bone. Tissue engineering scaffolds are used to improve the organism regeneration ability for this kind of injury, serving as biocompatible structures for cell viability and differentiation. Since biomechanical cues such as substrate stiffness, loading conditions and fluid permeation are fundamental for successful tissue repair, understanding how these features vary in the scaffold is of primary importance. Here we present a mathematical model based on porous media mechanics for the analysis of a tissue engineering scaffold. We consider a threelayered scaffold mimicking a complete osteochondral tissue and vary the mechanical properties of the intermediate layer over a physiological range. Our results show that the interstitial fluid pressure and the vertical component of the solid effective stress depend significantly on the stiffness and permeability of the intermediate layer under mechanical loading. By properly tuning these material properties, regimes of slow or fast temporal variations of mechanical stress can be obtained in the scaffold layer of interest.
\end{abstract}

